$15^{\text {th }}$ International Conference on

AEROSPACE SCIENCES \& AVIATION TECHNOLOGY,

$\boldsymbol{A S A T}$ - 15 - May 28 - 30, 2013, Email: asat@ mtc.edu.eg,

Military Technical College, Kobry Elkobbah, Cairo, Egypt,

Tel: +(202) 24025292 -24036138, Fax: +(202) 22621908

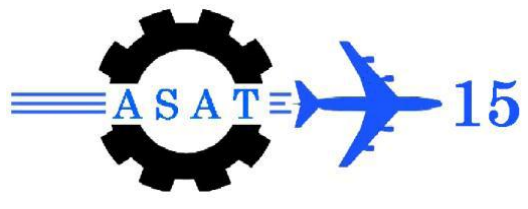

\title{
Application of Compressive Sensing in LFMCW Radar
}

\author{
\{S. G. Salem, F. M. Ahmed, M. H. Ibrahim, A. H. Elbardawiny\}*
}

\begin{abstract}
Compressive Sensing (CS) theory is a newly developed theory which combines the signal sampling and compression based on the sparsity characteristics of the signal. Applying CS theory in radar signal processing may lead to a reduction in sampling rate, complexity, power consumption, and cost. On the other hand, performance is a critical point to be considered.

In the present paper, an important question of the worthy of applying CS in the signal processing of Linear Frequency Modulated Continuous Wave (LFMCW) radar is considered. Two approaches of CS are considered; Nyquist rate based approach, and pseudo random based approach. The detection performance of LFMCW radar signal processor using CS based approaches is compared to the traditional one which is based on Fast Fourier Transform (FFT) through Receiver Operating Characteristics (ROC) curves. Comparative analysis between CS approaches and the traditional one regarding the performance and complexity is presented.
\end{abstract}

Keywords: Compressive sensing, FFT, and LFMCW.

\section{Introduction}

LFMCW radar is used to measure both the range and velocity of the target. LFMCW radar has the advantages of small size, weight, and simple implementation compared to pulse radar. To increase the range resolution (order of centimeter), high signal bandwidth is required and consequently, high sampling rate (order of Giga Hertz). The main bottleneck to implement the digital LFMCW radar receiver is shown in Figure (1). This is due to the fact that traditional Shannon Nyquist sampling is done by sampling at a rate greater than or equal to twice the highest frequency in a Signal of Interest (SoI)[1]. Furthermore, if it were possible to acquire wideband signals with conventional ADCs, it would cause a loss of data that needs to be processed [2, 3].

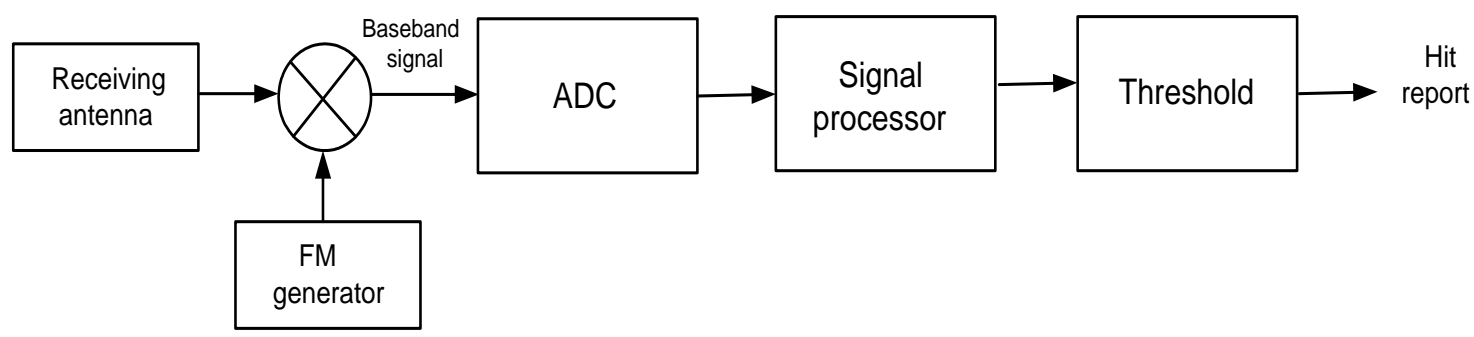

Figure (1): Block diagram of digital LFMCW radar receiver.

* Egyptian Armed Forces, Egypt. 
An efficient way to deal with these high data rates as well as the large amount of data it produces is the emerging field of Compressive Sensing (CS) [2]. CS theory states that, given certain circumstances, it is possible to reconstruct a signal sampled at a rate below the Nyquist rate $[4,5]$. A fundamental difference between CS and classical sampling is the manner in which the two frameworks deal with signal recovery. In the Shannon- Nyquist framework, signal recovery is achieved through sinc interpolation; a linear process that requires little computation and has a simple interpretation. In CS, however, signal recovery is achieved using nonlinear and relatively expensive optimization-based or iterative algorithms [6-7]. Thus, most of the CS literature has focused on improving the speed and accuracy of this process [8]. However, signal recovery is not actually necessary in many signal processing applications. Very often we are only interested in solving an inference problem (extracting certain information from measurements) or in filtering out information that is not of interest before further processing.

In the present paper, the application of various CS theory approaches in the signal processing of LFMCW radar is discussed and compared with the traditional one.

The rest of this paper is organized as follows: section 2 gives a survey on the principle of CS theory approaches. Section 3 describes the application of different CS approaches in LFMCW radar. Performance evaluation of LFMCW radar signal processing using CS approaches compared with the classical one is provided in section 4. Finally, the conclusion comes in section 5 .

\section{Compressive Sensing(CS) Theory}

Recently, the signal processing/mathematics community has seen a paradigmatic shift in the way information is represented, stored, transmitted and recovered [9]. This area is often referred to sparse representation and compressive sensing. Prerequisite of compressive sensing theory is that signal must be sparse or compressible. Under this prerequisite, compressive sensing projects high-dimensional signal into a lower dimensional projection signal. The process of compression and reconstruction of signal using compressive sensing (CS) theory is shown in Figure (2).

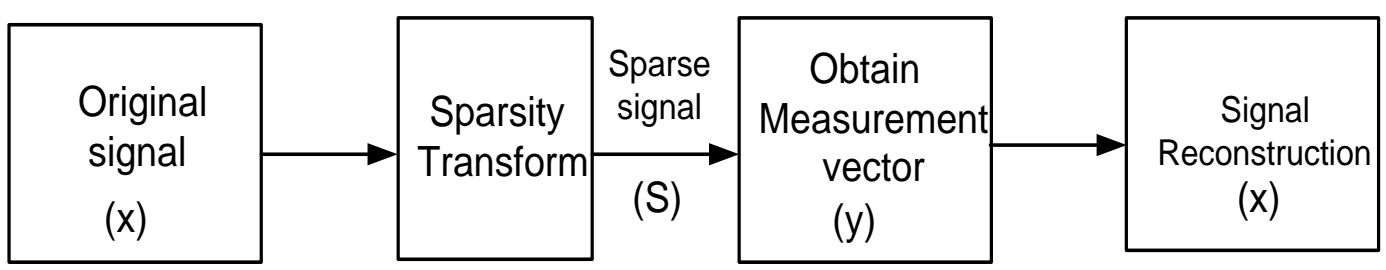

Figure (2): Block diagram of compressive sensing

The first step in Figure (2) is the sparsity transform of non sparse signal $x$. Suppose $S$ is onedimensional discrete-time signal with finite length and real value, which can be seen as a column vector in the space $R^{N}$. Known by the Matrix theory, any signal in the space $R^{N}$ can be represented by orthonormal basis $\left\{\mathrm{x}_{\mathrm{i}}\right\}$ where $\mathrm{i}$ from 1 to $N$ with linear form. Orthonormal basis $\left\{\mathrm{x}_{\mathrm{i}}\right\}$ can be rewritten as $X=\left[x_{1}, x_{2} \ldots x_{N}\right]$ in the form of Matrix. The spare signal $S$ in the space $R^{N}$ can be represented as: 


$$
S=\sum_{i=1}^{N} \xi_{i} x_{i}
$$

where $\xi_{\mathrm{i}}$ is coordinate of signal under the base vector $\mathrm{x}_{\mathrm{i}}$, whose specific form is $\xi_{i}=\left\langle S, x_{i}\right\rangle$. Equation (1) can be rewritten as:

$$
S=X \theta
$$

where $\theta=\left[\xi_{1}, \xi_{2}, \ldots . ., \xi_{N}\right]^{T}$.

It is clear that $\theta$ and $S$ are equivalent representations of the same signal, $x$. In other words, $S$ is time domain signal, and $\theta$ is the representation in the transformation domain.

Suppose $K$ be non-zero number of elements of $\theta$. If $K$ is smaller than $N$, it can be shown that the signal $S$ is sparse or compressible. Meanwhile, basis vector $\left\{x_{i}\right\}$ is the sparse basis of the signal $S[10]$.

The second step is to get $M$ observation values to ensure that the sparse signal $S$ or representation $\theta$ can be reconstructed. The process of observation is really the projection of sparse coefficients onto $M$ row vector $\left\{\varphi_{i}\right\}$ where i from 1 to $M$ of observation matrix $\Phi=\left[\phi_{1}, \phi_{2}, \ldots ., \phi_{M}\right]^{T}$, whose size is $M \times N$.

$M$ observation values can be obtained by inner product between $\theta$ and observation vector $\left\{\varphi_{i}\right\}$, which can be expressed as $y_{j}=\left\langle\theta, \phi_{j}\right\rangle, j=1, \ldots, M$.

Suppose observation vector $y=\left[y_{1}, y_{2}, \ldots ., y_{M}\right]^{T}$, the relationship between time domain signal $S$ and observation vector $y$ is given by[15]:

$$
y=\varphi \theta=\varphi X^{-1} S=\varphi X^{T} S=A^{C S} S
$$

where $A^{c s}$ is the measurement CS matrix.

As the dimension $M$ of observation vector is much smaller than the signal length $N$, the inverse problem of solving equation (3) is an ill-posed problem, and so the sparse signal $S$ or sparse coefficients cannot be directly observed by $M$ observation values [15].

Through sparse signal decomposition in the theory of sparse decomposition algorithm, by solving the inverse problem of equation (3), sparse coefficients $\theta$ can be obtained, and the signal $S$ can be obtained. To ensure the convergence of the algorithm, the observation matrix in Equation (3) must meet the Restricted Isometry Property (RIP) criteria [11]. Also, If $\phi$ is sufficiently "incoherent," then the information of $S$ will be embedded in $y$ such that it can be perfectly recovered with high probability. Current reconstruction methods use greedy algorithms such as Orthogonal Matching Pursuit (OMP) [7], Basis Pursuit (BP) [5, 6], Regularized Orthogonal Matching Pursuit (ROMP) [12], Complex Approximate Message Passing (CAMP) [13] and the Manhattan_norm, $\ell_{1}[14]$.

In the present work, the Manhattan $\ell_{1} \_$norm, and Complex Approximate Message Passing (CAMP) algorithms are considered. These two algorithms represent the earliest and the recent algorithms used in literature [13].

There are two approaches for using CS. The first one is based on sampling the original signal with Nyquist rate. The second one is based on sampling the original signal with random 
sampling function. In the following subsections, discussion of these two approaches is presented.

\subsection{Nyquist Rate Based CS}

First, to obtain sparse signals, the sparse matrix generation is based on the characteristics of the original signal. According to the characteristics of the sinusoidal signal of LFMCW radar, sparse atomic database can be created using Fourier transformation [18].

The signal will be acquired with a rate depends on Shannon theory and then transformed by Fourier transformation to generate sparse signal. These sparse signals is used to generate a compressed vector, $y_{m}$, after multiplying with measurement matrix, $\Phi$, as shown in Figure (3). The original sparse signal can be reconstructed from the compressed measurement using two approaches; $\ell_{1}$-norm and CAMP algorithms.

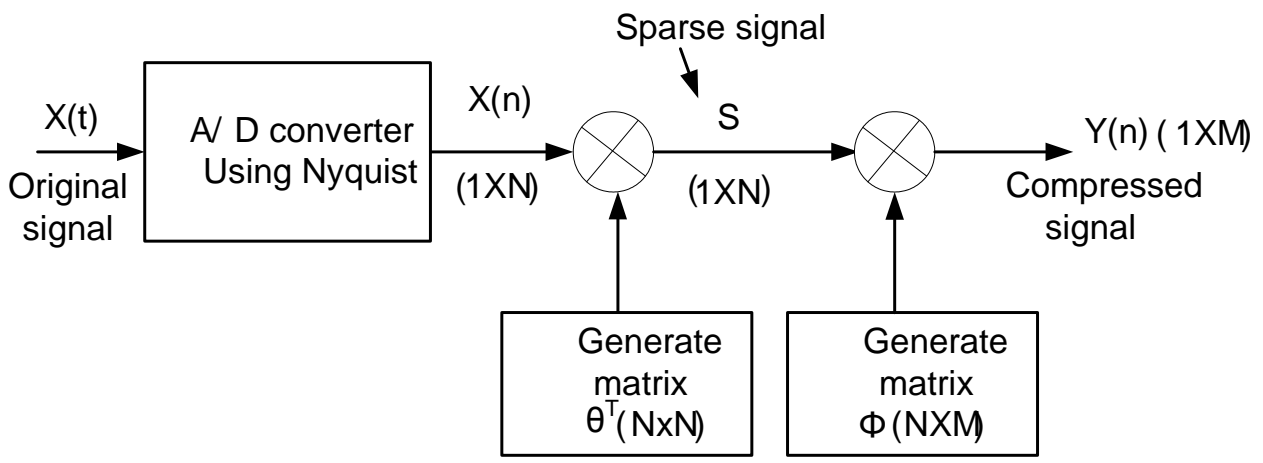

Figure (3): Measurement matrix and compressed signal generation.

\subsection{1 $\ell_{1}$-norm Reconstruction Algorithm}

In the compressed sensing theory, because the number of observations $M$ is much smaller than the signal length $N$ where $M \geq K \log (N / K)$ [2], thus we are faced with solving indefinite equations. Under the prerequisites that signal $S$ is sparse, the problem of solving Equation (3) can be transformed into minimizing $\ell_{1}$ norm. The minimization using $\ell_{1}$-norm method, also known as Least Absolute Values Method (LAVM), not always give unique solution and there may be several solutions. Also, the solution of $\ell_{1}$-norm method is not generally obtained directly, but iteratively. Calculations are made according to Equation (4). More details about $\ell_{1}$-norm can be found in [16]

$$
y=\min \left\|X^{T} S\right\|_{1}
$$

\subsubsection{CAMP Reconstruction Algorithm}

The Approximate Message Passing (AMP) is an iterative algorithm used for solving Equation (3) in the presence of real valued signals and measurements. Recently AMP has been extended to the case of signals in the complex domain, resulting in the Complex AMP (CAMP). It is a recently developed sparse signal recovery algorithm that delivers excellent recovery performance, exhibits fast convergence at low computational complexity per iteration, while requiring low arithmetic precision, compared to $\ell_{1}$-norm method [13].

The estimated signal is firstly initialized as 


$$
\mathrm{z}^{\mathrm{o}}=\mathrm{y}
$$

where $\mathrm{z}^{\mathrm{o}}$ is the measurement matrix at certain iteration.

The CAMP algorithm computes the reconstructed signal according to the following equations [13]:

$$
\begin{aligned}
& \tilde{x}^{i}=A^{i} Z^{i-1}+\hat{x}^{i-1} \\
& T=\operatorname{average}\left(\tilde{x}^{i}\right)
\end{aligned}
$$

where $\tilde{x}^{i}$ is the non sparse estimation of the signal, $\mathrm{x}$, and $\mathrm{T}$ is the threshold of the estimated non sparse signal.

\subsection{Pseudo Random Based CS}

The process of acquiring signals compressively using the random demodulator is shown in Figure (4) $[17,18]$. The random demodulator scrambles the received signal by mixing it with a pseudorandom sequence. The scrambled signal is then sampled at a rate below the Nyquist rate of the SoI. The receiver operates with pseudo random method differs from traditional CS as it does not reconstruct the received signal, so processing is done directly on the compressed samples. This approach avoids the computationally intensive non linear reconstruction algorithms and results in less data having to be processed.

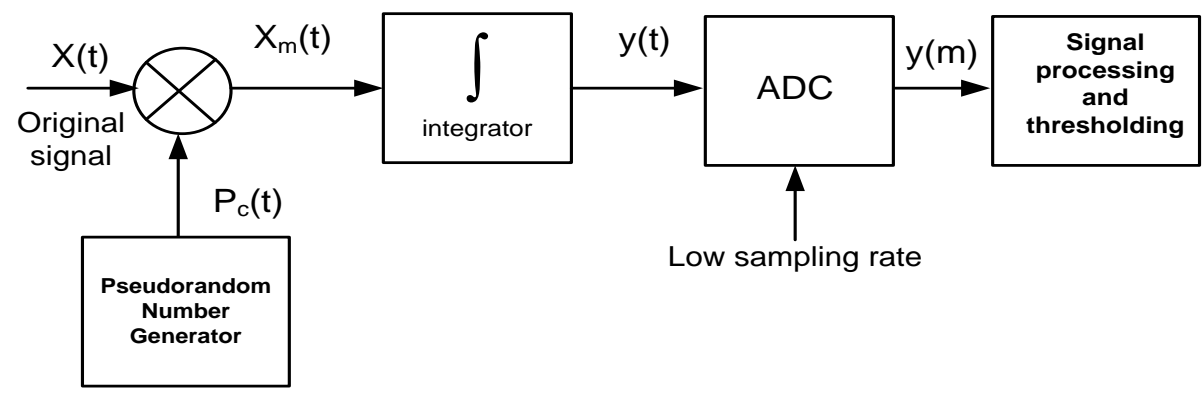

Figure (4): Random demodulator.

The received wideband signal, $x(t)$, is mixed with a random chipping sequence, $P_{c}(t)$, consisting of pseudorandom (0 or 1$)$ values [19].

The mixed signal is then summed for $t_{L}$ seconds using an integrate-and-dump circuit, and the summed value is sampled using a low rate ADC. The time for which the signal is summed, $t_{L}$, is related to the decimation/sub-sampling factor $L$ by the equation

$$
t_{L}=L T_{N}
$$

where $T_{N}=1 / f_{N}$ and $f_{N}$ denotes the Nyquist frequency of the received signal. The effective sampling rate is thus given by

$$
f_{S}=\frac{f_{N}}{L}
$$

The resulting sampled signal $y(m)$, is a discrete vector with length $M$, where:

$$
M=\frac{N}{L}
$$




\section{Compressive Sensing LFMCW Radar}

Traditional LFMCW radar theory assumes that the measured return signal after mixing is a sinusoidal signal whose frequency is a function of the distance of targets. The traditional processing of this signal depends mainly on applying FFT to extract range information [20]. To achieve high range resolution, a wide bandwidth should be used implies high sampling rate.

In the present paper, to get the benefit of CS theory in reducing sampling rate and complexity, different CS approaches are applied on LFMCW radar signal processing and compared with the traditional one as shown in Figure (5).

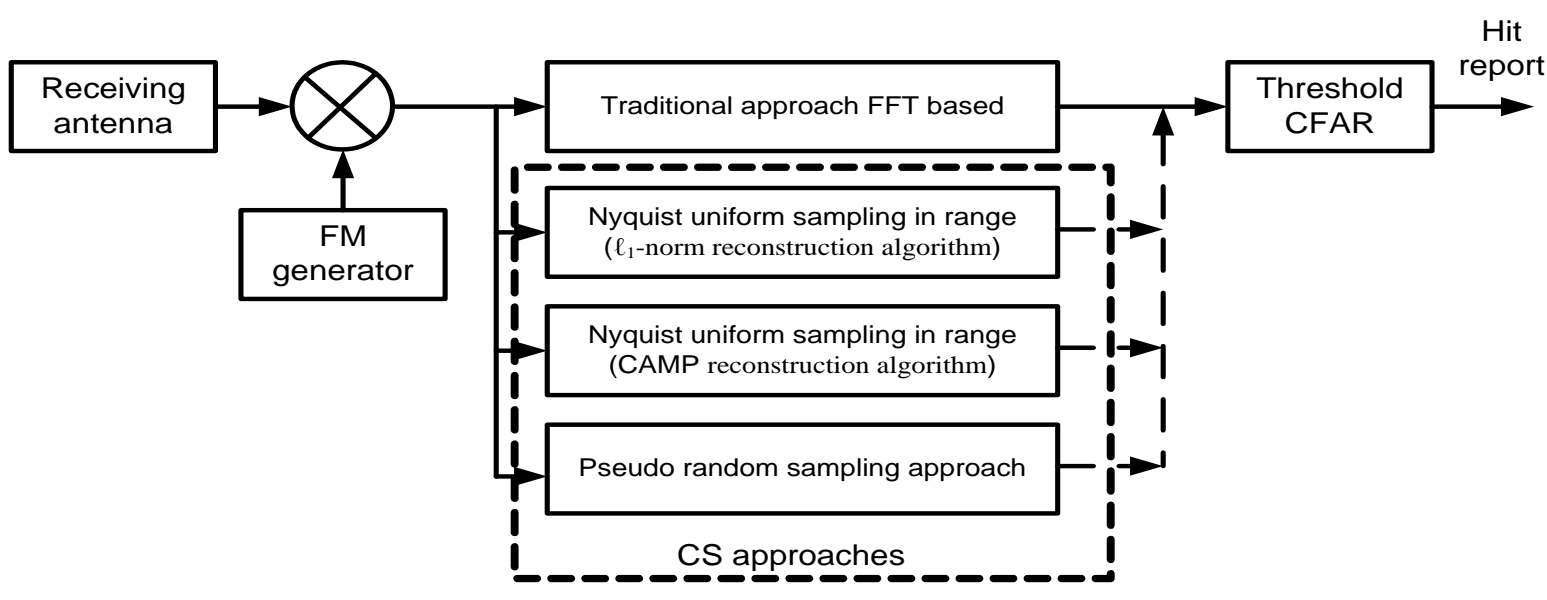

Figure (5): Block diagram of LFMCW radar receiver with applying different CS approaches.

Since the demodulated radar signal is sinusoidal, so, it is not sparse. To apply Nyquist rate CS based approaches, it is required to transform this signal into sparse signal. Transforming this signal into sparse one is equivalent to obtaining its FFT. Then, the obtained sparse signal should be compressed to obtain a measurement vector. This vector is then reconstructed to the sparse form again.

If $\ell_{1}$-norm algorithm is applied in reconstruction, no improvement in performance is expected [13]. On the other hand, extra calculation is imposed. The CAMP reconstruction algorithm is expected to give an improvement in the output Signal to Noise Ratio (SNR) compared to original signal [13]. Also, less calculations and complexity compared to $\ell_{1}$-norm method is expected. Applying the pseudo random CS based approach takes place directly on the demodulated radar signal. It should lead to a reduction in sampling rate and complexity [18]. On the other hand, a reduction in performance is expected.

To validate the previous discussion, it is essential to compare between traditional and CS based LFMCW radar signal processing in terms of performance and complexity. The detection comparison shall be achieved through the ROC curves. The complexity comparison shall be achieved through the time and number of calculations. These comparisons may give some guidelines for applying CS in LFMCW radar. 


\section{Simulation Results}

Simulation results are obtained based on Monte-Carlo trials using MATLAB package.

For the traditional FFT based method or the Nyquist rate based CS approaches, the demodulated LFMCW radar signal is assumed to be acquired with the Nyquist rate. The number of samples to be processed is assumed to be 512 samples.

For traditional FFT approach, the FFT algorithm is applied on the total 512 samples.

For the Nyquist rate based CS approaches, the transform matrix is generated to be $256 \mathrm{X} 512$ resulting in a measurement vector of 256 samples.

For the pseudo random based CS approach, the pseudo random generator selects 256 random samples from the original 512 samples.

The thresholding process is achieved through realizing a Cell- Averaging Constant False Alarm Rate (CA-CFAR) processor with total window size of 16 cells [21].

Figure (6) shows the relation between the probabilities of false alarm for Nyquist rate based approaches $\left(\ell_{1}\right.$-norm and CAMP), Pseudo random based approach, and traditional FFT-based approach at different CFAR threshold gain. The CAMP approach gives the ideal $P_{f a}$ which is zero. This is because that the CAMP algorithm itself contains a threshold function which does not permit a false alarm at the output. This leads to a reduction in complexity by omitting the CFAR processor for the signal processing if this method is used.

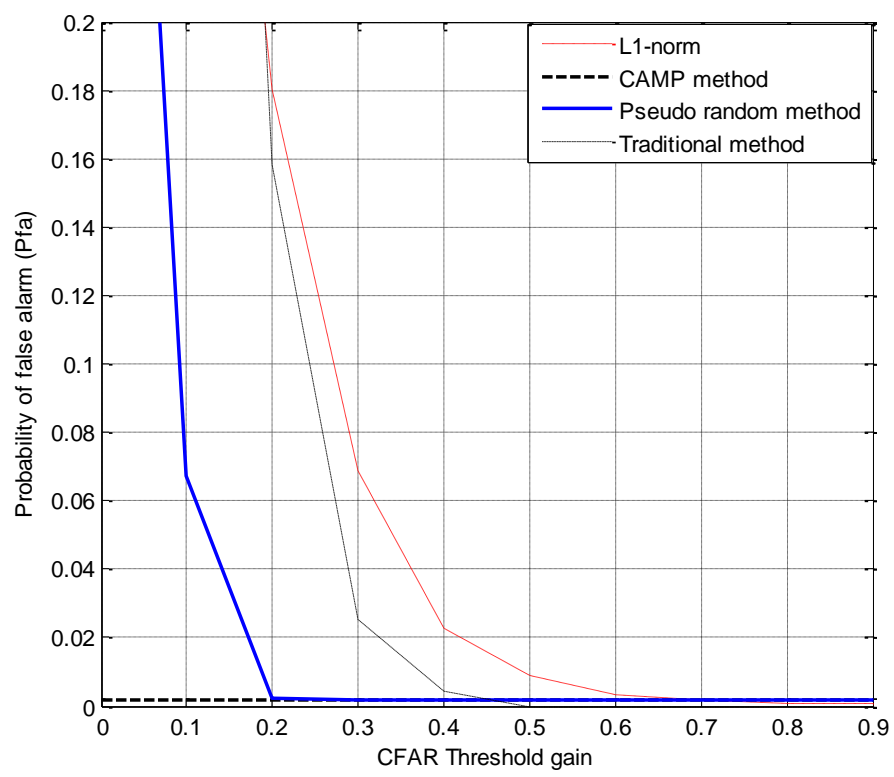

Figure (6): The relation between probabilities of false alarm of LFMCW radar receiver using Nyquist-based ( $\ell 1$ norm,CAMP), Pseudo random and traditional approaches.

Regarding to the detection performance Figures (7),(8), and (9) show the ROC curves for range processing using Nyquist rate based approach ( $\ell_{1}$-norm and CAMP), Pseudo random based approach and FFT-based LFMCW approach for single target detection at $\mathrm{P}_{\mathrm{fa}}$ of $10^{-3}$, 
$10^{-4}$ and $10^{-5}$ respectively. With respect to CAMP algorithm, the detection does not depend on $\mathrm{P}_{\mathrm{fa}}$ of CFAR. It depends only on the SNR of the received signal. Controlling the false alarm probability for the CAMP algorithm is achieved by controlling the value of the internal threshold of the CAMP algorithm.

It is clear that the detection performance due to applying the CAMP algorithm in LFMCW radar is better than the other approaches.

The $\ell_{1}$-norm based approach gives the worst detection performance. The pseudo random approach gives approximately a lower $10 \%$ detection than the traditional FFT.

Regarding to the complexity, the $\ell_{1}$-norm approach gives the highest complexity and time of calculation. The CAMP approach gives less complexity and time of calculation with respect to $\ell_{1}$-norm, but still gives more complexity of calculation with respect to the traditional one.

The pseudo random approach gives the less complexity of calculation with respect to other approaches.

From the obtained results, it is not recommended to apply $\ell_{1}$-norm based CS approach to LFMCW radar since it gives the worst detection and the highest complexity. Using the CAMP approach gives the best detection performance with an extra calculation complexity which may be compensated for by removing the CFAR processor and by using advanced tools in implementation [22]. The pseudo random approach gives a considerable performance compared to the traditional one with a reduction in sampling rate and complexity of calculation.

Table (1) gives a simple comparison between the discussed approaches with respect to detection performance at certain SNR of $10 \mathrm{~dB}$ and $\mathrm{P}_{\mathrm{fa}}$ of $10^{-4}$. Table (2) compares between the discussed approaches with respect to the complexity of the used algorithms, time of calculations (number of iterations) and the used sampling rate. According to these two tables, one should compromise between performance and the extra amount of complexity according to his/her requirements.

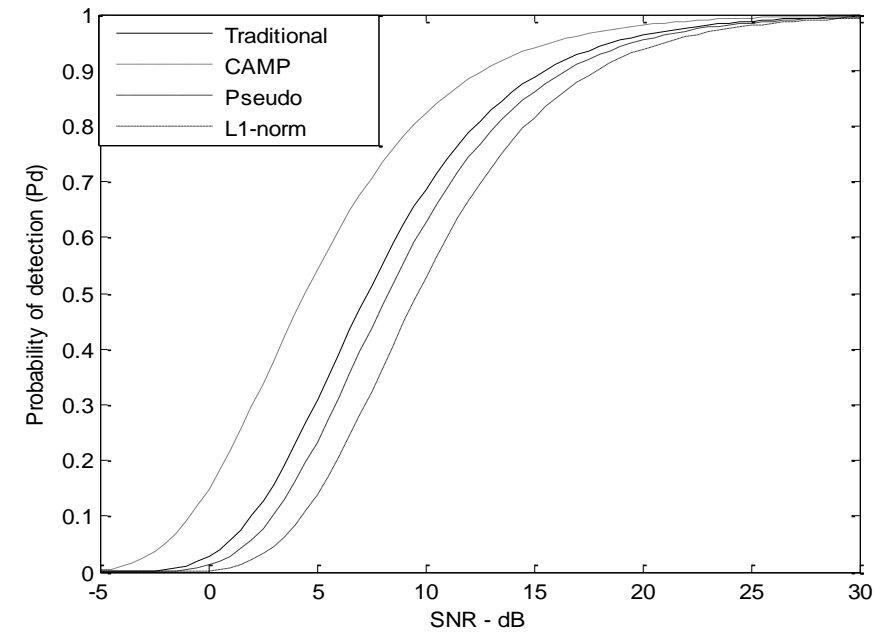

Figure (7): ROC of the Nyquist-based LFMCW radar receiver $\left(\ell_{1}\right.$ norm and CAMP) compared to Pseudo random-based and traditional FFT-based at $\mathbf{P}_{\mathrm{fa}}=10^{-3}$. 


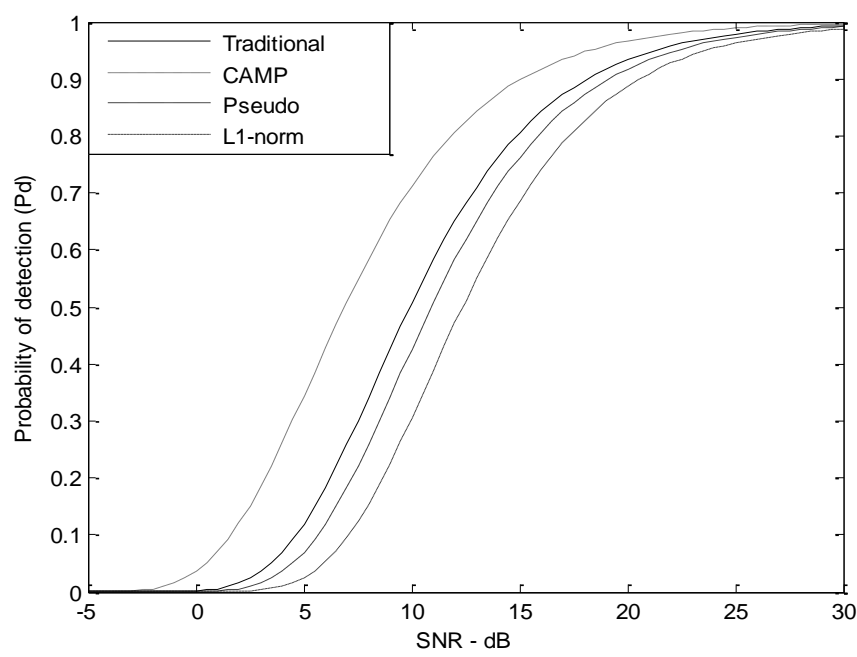

Figure (8): ROC of the Nyquist-based LFMCW radar receiver $\left(\ell_{1}\right.$ norm and CAMP) compared to Pseudo random-based and traditional FFT-based at $P_{\mathrm{fa}}=\mathbf{1 0}^{-4}$.

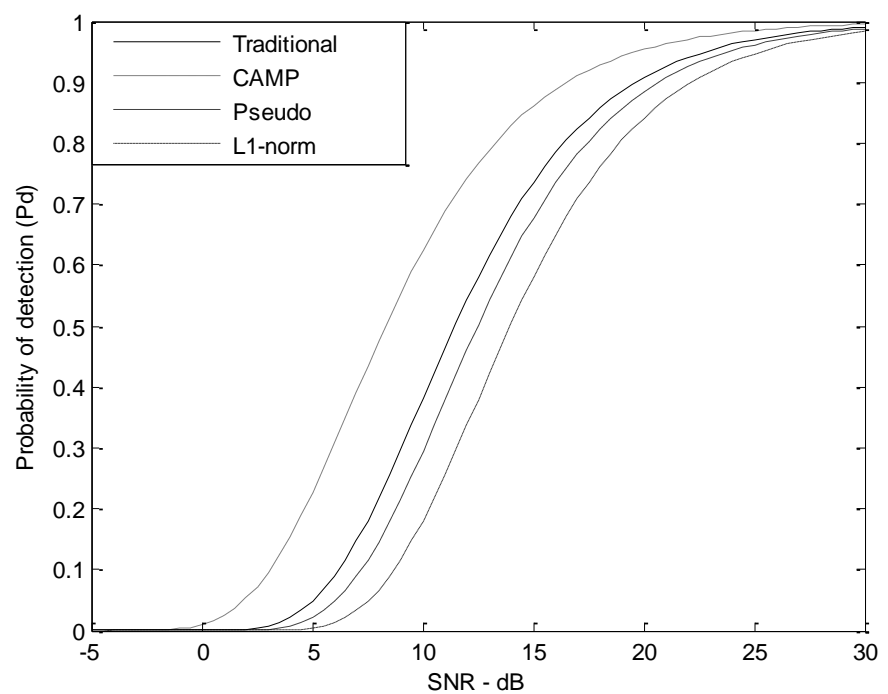

Figure (9): ROC of the Nyquist-based LFMCW radar receiver( $\ell_{1}$ norm and CAMP) compared to Pseudo random-based and traditional FFT-based at $P_{\mathrm{fa}}=10^{-5}$.

Table (1) Comparison among CS approaches and traditional one in terms of detection performance at $S N R=10 \mathrm{~dB}$ and $P_{\mathrm{fa}}=10^{-4}$

\begin{tabular}{c|c|c}
\hline \hline Order (high to low) & Approach & Detection performance $\left(\mathrm{P}_{\mathrm{d}}\right) \%$ \\
\hline 1 & Nyquist rate $($ CAMP) & 79 \\
\hline 2 & Traditional & 52 \\
\hline 3 & Pseudo random & 49 \\
\hline 4 & Nyquist rate $\left(\ell_{1}\right.$ norm) & 30 \\
\hline
\end{tabular}


Table (2) Comparison among CS approaches and traditional one in terms of complexity, time of calculations (number of iterations) and sampling rate.

\begin{tabular}{c|c|c|c|c}
\hline $\begin{array}{c}\text { Order (low } \\
\text { to high) }\end{array}$ & Approach & Complexity & Time(iterations) & Sampling rate \\
\hline 1 & Pseudo random & $\begin{array}{c}\text { PNG + smoothing } \\
\text { filter + ADC + 256 } \\
\text { points FFT }\end{array}$ & -- & $\mathrm{f}_{\mathrm{s}} / 2$ \\
\hline 2 & Traditional & $\begin{array}{c}\text { ADC + 512 points } \\
\text { FFT }\end{array}$ & -- & $\mathrm{f}_{\mathrm{s}}$ \\
\hline 3 & $\begin{array}{c}\text { Nyquist rate } \\
\text { (CAMP) }\end{array}$ & $\begin{array}{c}\text { ADC + sparse } \\
\text { transform + } \\
\text { reconstruction } \\
\text { algorithm }\end{array}$ & 10 & $\mathrm{f}_{\mathrm{s}}$ \\
\hline 4 & $\begin{array}{c}\text { Nyquist rate } \\
\left(\ell_{1} \text { norm }\right)\end{array}$ & $\begin{array}{c}\text { transform }+ \\
\text { reconstruction } \\
\text { algorithm }\end{array}$ & 32 & $\mathrm{f}_{\mathrm{s}}$ \\
\hline \hline
\end{tabular}

\section{Conclusion}

In this paper, the possibility of applying CS theory in the signal processing of LFMCW radar is introduced. Different CS approaches such as the Nyquist rate $\left(\ell_{1}\right.$-norm, CAMP), pseudo random based and traditional FFT based approaches have been applied and compared with respect to detection performance through ROC curves and complexity of calculations. The Nyquist rate based CS $\left(\ell_{1}\right.$-norm) approach has been found to give the worst detection performance and the highest calculation complexity. The Nyquist rate based CS (CAMP) approach has been found to give the highest detection performance and an extra amount of calculations. These extra calculations can be compensated by removing the CFAR processor and using advanced implementation tools. The pseudo random approach has been found to give a less but good detection performance with respect to the traditional one with the least calculation complexity.

Finally, if performance is considered, the CAMP is the choice. If complexity is considered, the pseudo random is the best.

\section{References}

[1] B.Le, T.Rondeau, J.Reed, and C.Bostian, "Analog-to-Digital converters", Signal Processing Magazine, vol.22, no.6,pp. 69-77, 2005.

[2] R. G. Baraniuk, "More is less: Signal processing and the data deluge," Science, vol. 331, no. 6018, pp. 717-719, Feb 2011.

[3] J. N. Laska, W. F. Bradley, T. W. Rondeau, K. E. Nolan, and B. Vigoda, "Compressive sensing for dynamic spectrum access networks: Techniques and tradeoffs," in IEEE Int. Symposium on Dynamic Spectrum Access Networks (DySPAN), 2011.

[4] E. Candes, "Compressive sampling," in Proc. Int. Congress of Mathematics, pp. 1433 1452, Aug. 2006.

[5] D. Donoho, "Compressed sensing," Information Theory, IEEE Transactions on, vol. 52, no. 4, pp. 1289-1306, 2006. 
[6] E. Cand'es, J. Romberg, and T. Tao, "Robust uncertainty principles: Exact signal reconstruction from highly incomplete frequency information," IEEE Trans. Inform. Theory, vol. 52, no. 2, pp. 489-509, 2006.

[7] J. Tropp and A. Gilbert, "Signal recovery from partial information via orthogonal matching pursuit," IEEE Trans. Inform. Theory, vol. 53, no. 12, pp. 4655-4666, Dec.2007.

[8] D. Needell and R. Vershynin, "Uniform uncertainty principle and signal recovery via regularized orthogonal matching pursuit," Found. Comput. Math., vol. 9, no. 3, pp. 317-334, 2009.

[9] J. A. Tropp, "Greed is good: Algorithmic results for sparse approximation," IEEE Trans. Inf. Theory, vol. 50, no. 10, pp. 2231-2242, Oct.2004.

[10] Jing Li, Shunsheng Zhang, Junfei Chang, "Two-Dimensional Random Sparse Sampling for High Resolution SAR Imaging Based on Compressive Sensing”, IEEE Journal of selected Topics in signal proc., vol. 1, no. 2, pp. 653-673, 2012.

[11] Zhu Lei, Qiu Chunting, "Application of compressed sensing theory to radar signal processing", IEEE Journal of selected Topics in signal proc.,2010.

[12] E. Cand'es, "The retricted isometry property and its implications for compressed sensing", C.R. Acad. Sci. Paris ,Ser. I, 346, pp. 589-592, 2008.

[13] D. Needell and R. Vershynin, "Uniform uncertainty principle and signal recovery via regularized orthogonal matching pursuit," July 2007.

[14] Laura Anitori, Matern Otten, Peter Hoogeboom, Arian Maleki ,Richard G. Baraniuk, "Compressive CFAR Radar Detectors", IEEE Journal of selected Topics in signal proc.,pp.0320-0325,2012.

[15] R. G. Baraniuk, “Compressive sensing”, Lecture notes, IEEE Signal Proc. Magazine, pp.118-124, 2007.

[16] M.A.Davenport, T. Boufounos, Michael B. Wakin, and Richard G. Baraniuk, "Signal Processing with Compressive Measurements", IEEE Journal of selected Topics in signal proc., vol. 4,no. 2, pp. 445-450, 2010.

[17] Sebahattin Bektas and Yasemin sisman, "The comparison of L1 and L2-norm minimization methods", International Journal of the Physical Sciences Vol. 5(11), pp. 1721-1727, 18 Sep., 2010.

[18] M. A. Davenport, S. R. Schnelle, J. P. Slavinsky, R. G. Baraniuk, M. B. Wakin, and P. T. Boufounos, "A wideband compressive radio receiver," in Military Communications Conference (MILCOM), San Jose, California, Oct. 2010.

[19] J. Tropp, J. Laska, M. Duarte, J. Romberg, and R. Baraniuk, "Beyond nyquist: Efficient sampling of sparse bandlimited signals," Information Theory, IEEE Transactions on, vol. 56, no. 1, pp. 520 -544, Jan. 2010.

[20] Sameh G.Salem, Fathy M. Ahmed, Mamdouh H.Ibrahim, Abdel Rahman H. Elbardawiny, "A New Approach of LFMCW Radar using Wigner-Ville Distribution," 8th International Conference on Electrical Engineering ICEENG, Egypt, (May 2012).

[21] Assem Moustafa, Fathy M.Ahmed,K.H.Moustafa,Y.Z.Halwagy, "A New CFAR processor Based on Guard Cells Information," IEEE radar conference, Atlanta, USA, pp.0133-0137,May 2012.

[22] Lin Bai, Patrick Maechler, Michael Muehlberghuber, and Hubert Kaeslin, "High-Speed Compressed Sensing Reconstruction on FPGA Using OMP and AMP," IEEE journal on Emerging and Selected Topics in Circuits and Systems, 2012. 